\title{
ANÁLISE COMPARATIVA DO TEOR DE FLAVONOIDES E DA ATIVIDADE ANTIOXIDANTE DE EXTRATOS DE BACCHARIS UNCINELLA OBTIDOS POR EXTRAÇÃO SUPERCRÍTICA E MACERAÇÃO.
}

\author{
M.E.Sanvido ${ }^{1}$; J.M. Scopel ${ }^{1}$; E. Cassel ${ }^{1}$; A.M. Lucas ${ }^{1}$ \\ ${ }^{1}$ Pontifícia Universidade Católica do Rio Grande do Sul \\ E-mail para contato: maria.sanvido@acad.pucrs.br
}

\begin{abstract}
RESUMO - O gênero Baccharis compreende cerca de 500 espécies distribuídas pelo continente americano. Os principais compostos relatados em extratos não voláteis do gênero Baccharis são os compostos fenólicos, dentre eles, os flavonoides e terpênicos. Suas principais propriedades biológicas são as atividades antioxidante, antibacteriana e anti-inflamatória. Neste estudo foram empregadas duas técnicas extrativas: extração supercrítica e maceração seguida de partição líquido-líquido. O objetivo do trabalho foi avaliar a atividade antioxidante e o teor flavonoides no extrato, comparando as amostras oriundas da maceração e da extração supercrítica. Foram utilizadas, como matéria prima, as partes aéreas de Baccharis uncinella para ambas as técnicas de extração. A extração supercrítica foi realizada em temperatura de $60{ }^{\circ} \mathrm{C}$ e pressões de 150 e 200 bar. $\mathrm{O}$ cossolvente etanol foi adicionado a fim de aumentar a polaridade do sistema e melhorar a seletividade da extração em relação a compostos fenólicos. A extração convencional foi realizada com metanol e a planta foi macerada por 10 dias, posteriormente o mesmo foi particionado com solventes de polaridades diferentes: n-hexano, diclorometano e acetato de etila. Os resultados obtidos indicam que o extrato metanólico particionado com acetato de etila foi o que apresentou a melhor atividade antioxidante $\left(\mathrm{CE}_{50}=11,80\right)$ e maior percentual de flavonoides na fração $(5,28 \%)$.
\end{abstract}

\section{INTRODUÇÃO}

O gênero Baccharis, pertencente à família Asteraceae, compreende aproximadamente 500 espécies, todas americanas, onde aproximadamente 120 estão localizadas no Brasil (Budel et al., 2005). Entre os principais compostos encontrados em extratos de plantas deste gênero estão os compostos terpênicos e os compostos fenólicos. Destacam-se os flavonoides, que são amplamente empregados nas indústrias de alimentos, farmacêutica e cosmética por estarem relacionados às atividades antimicrobianas, antioxidantes e anti-inflamatórias (Ascari et al., 2012; Piantino et al., 2008; Vila, 2006).

Diferentes técnicas são utilizadas para a obtenção de extratos não voláteis de material vegetal, sendo que tradicionalmente o método empregado é a maceração da planta com solvente orgânico (Biesaga et al., 2011). Quando o interesse é a obtenção de compostos polares, o metanol é o solvente mais utilizado, pois consegue extrair um maior número de compostos (Cechinel et al, 1998). A partição do extrato macerado como operação posterior ao 
processo de extração também é fruto de estudos, pois a partir da aplicação da mesma é possível obter frações do extrato bruto com polaridades diferentes (Cechinel et al., 1998). Como alternativa ao processo tradicional, a extração supercrítica é uma operação unitária que tem ganhado destaque no processo extrativo de compostos bioativos a partir de matrizes vegetais (Huang et al., 2012). Entre as vantagens relacionadas ao seu uso, principalmente quando o solvente utilizado é o $\mathrm{CO}_{2}$, é o fato de ser considerada uma tecnologia limpa (Cassel et al., 2008). $\mathrm{O} \mathrm{CO}_{2}$ é o solvente mais utilizado por ser não inflamável, não tóxico, não corrosivo e atingir facilmente as condições de temperatura e pressão supercríticas (Cassel et al., 2008; Huang et al., 2012). Com o objetivo de alterar a polaridade do solvente supercrítico, são incorporados cossolventes, promovendo assim o aumento do poder de solvência e a seletividade do processo de extração supercrítica (Cassel et al., 2008).

Para a análise de extratos não voláteis, destacam-se as técnicas cromatográficas, como a cromatografia em camada delgada e técnicas espectrofotométricas de determinação da concentração de compostos específicos, como a análise de flavonoides (Funari e Ferro, 2006). Análises de atividades biológicas também são muito utilizadas, dentre elas, a análise de atividade antioxidante, que indica o potencial de proteção a oxidação encontrado em determinados compostos (Oliveira et al., 2003).

A busca de extratos e frações de Baccharis uncinella com elevada atividade antioxidante e alta concentração de flavonoides, assim como a avaliação e a seleção do processo extrativo que potencialize a obtenção de compostos com estas características, concentram os objetivos deste estudo.

\section{MATERIAIS E MÉTODOS}

\subsection{Coleta do material vegetal}

O material vegetal utilizado nos experimentos foi coletado no Centro de Pesquisa e Conservação da Natureza Pró-Mata (PUCRS), localizado em São Francisco de Paula, RS. Para os experimentos de extração foram utilizadas as partes aéreas da planta. A planta foi seca em estufa (Tecnal) a uma temperatura de $40^{\circ} \mathrm{C}$ e moída em moinho de facas (Skymsen).

\subsection{Extração supercrítica}

As extrações dos compostos não voláteis de Baccharis uncinella foram realizadas na unidade piloto de extração supercrítica na temperatura de $60^{\circ} \mathrm{C}$ e nas pressões de 150 e 200 bar, considerando $50 \mathrm{~g}$ das partes aéreas secas e moídas da planta. O solvente empregado foi $\mathrm{CO}_{2}(99,9 \%$ de pureza - AirProducts). Para os experimentos realizados com cossolvente, foi selecionado etanol para ser adicionado ao $\mathrm{CO}_{2}$, com o objetivo de aumentar a polaridade do solvente supercrítico. A vazão de $\mathrm{CO}_{2}$ foi de $700 \mathrm{~g} / \mathrm{h}$. No caso dos experimentos com adição de cossolvente, a concentração de etanol foi de $10 \%$ em massa em relação à vazão do $\mathrm{CO}_{2}$.

\subsection{Maceração e Partição}

Para a maceração das partes aéreas moídas e secas de Baccharis uncinella foi utilizado metanol (Merck) como solvente. A massa de material vegetal empregada foi de $50 \mathrm{~g}$, assim como no processo com extração supercrítica, e o volume de solvente foi de $500 \mathrm{~mL}$, por um período de 10 dias. $\mathrm{O}$ extrato obtido foi seco em estufa à temperatura controlada $\left(35^{\circ} \mathrm{C}\right)$. De 

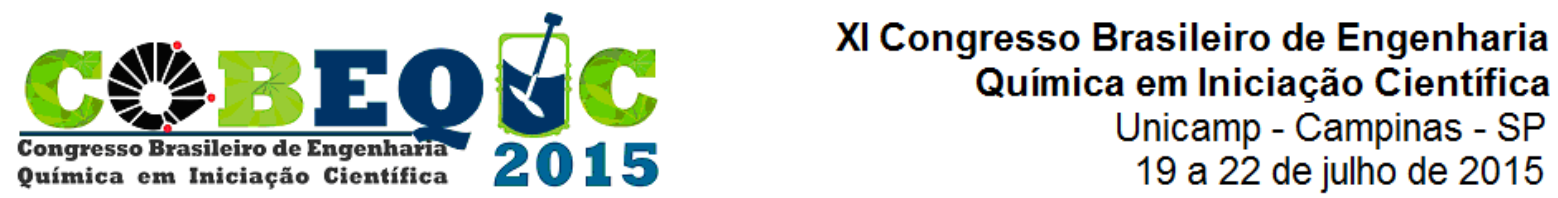

posse do extrato seco, o mesmo foi diluído em $100 \mathrm{~mL}$ de água destilada. Esta solução foi particionada com a adição sequencial de $50 \mathrm{~mL}$ dos seguintes solventes: n-hexano (Merck), diclorometano (Merck) e acetato de etila (Merck).

\subsection{Análises}

Cromatografia em Camada Delgada: A análise por cromatografia em camada delgada (CCD) foi realizada em placas de sílica gel Alugram ${ }^{\circledR}$ Xtra SIL G/UV ${ }_{254}$ (Macherey-Nagel) de $20 \mathrm{~cm} \times 20 \mathrm{~cm}$, usando como fase móvel diclorometano PA (Merck). As placas foram visualizadas em lanterna de luz UV (Boiton) com comprimento de onda de $\lambda=365 \mathrm{~nm}$. Após a visualização na luz UV, as placas foram reveladas com DPPH, para avaliar a atividade antioxidante, vanilina sulfúrica e cloreto de alumínio, para avaliar a presença de compostos fenólicos e flavonoides, respectivamente.

Análise de Atividade Antioxidante: A análise da atividade antioxidante dos extratos foi realizada através do método de captura do radical livre DPPH, proposto por Brand-Williams (1995). As amostras dos extratos e das frações particionadas permaneceram reagindo por 60 minutos em um local protegido da luz. A leitura das absorbâncias foi realizada em espectrofotômetro (Biospectro SP-220) no comprimento de onda de $\lambda=515 \mathrm{~nm}$.

Determinação de Flavonoides Totais: $\mathrm{O}$ método da reação com $\mathrm{AlCl}_{3}$ (Funari e Ferro, 2006) foi utilizado para a determinação de flavonoides totais. O tempo de reação foi de 30 minutos e a leitura da absorbância das amostras foi realizada no comprimento de onda $\lambda=425 \mathrm{~nm}$ em espectrofotometro (Biospectro SP-220). O percentual de flavonoides foi determinado em relação à concentração de quercitina.

\section{RESULTADOS E DISCUSSÕES}

\subsection{Cromatografia em Camada Delgada (CCD)}

A imagem da CCD gerada em luz UV no comprimento de onda de $\lambda=365 \mathrm{~nm}$ é apresentada na Figura 1a, enquanto que as CCDs reveladas com DPPH, vanilina sulfúrica e cloreto de alumínio são apresentadas na Figura 1b, 1c e 1d, respectivamente.

Figura 1-Imagem das CCDs (a) gerada em luz UV para $365 \mathrm{~nm}$; revelada com DPPH (b), vanilina sulfúrica (c) e cloreto de alumínio (d) dos extratos e frações de $B$. uncinella

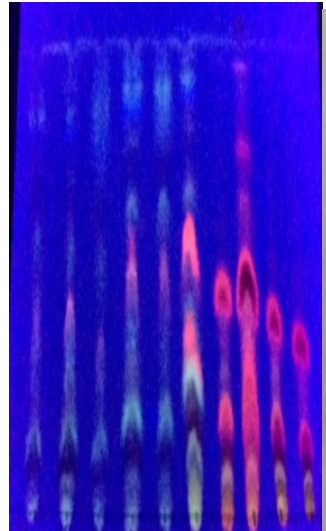

(a)

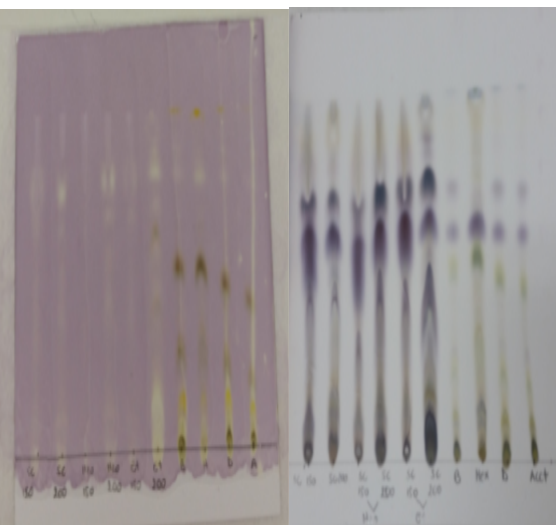

(b) (c)

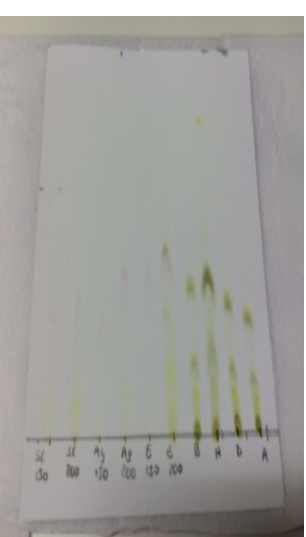

(d) 

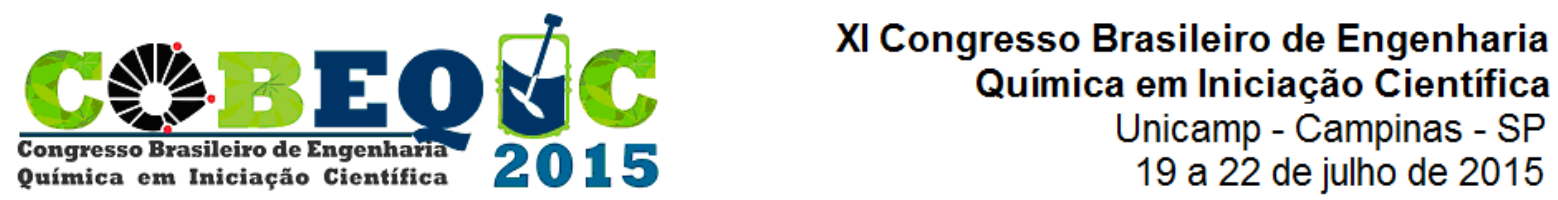

Avaliando a imagem da Figura la se percebe a presença de flavonoides pela fluorescência azul e amarela para todos os extratos e frações analisados. Na CCD revelada com DPPH (Figura 1b) foi possível verificar que todos os extratos apresentaram atividade antioxidante pela coloração amarela que adquiriram. Na CCD revelada com vanilina sulfúrica (Figura 1c), observa-se a presença de compostos fenólicos, tanto nos extratos supercríticos como nos extratos oriundos da maceração, visto que se visualiza a presença de manchas amarela/alaranjada. Resultados semelhantes são observados na CCD revelada com cloreto de alumínio (Figura 1d), onde se nota a presença de manchas amarelas indicativas de flavonoides.

\subsection{Análises de atividade antioxidante e flavonoides}

Nas Tabelas 1 e 2 são mostrados os resultados das análises antioxidantes e o percentual de flavonoides das extrações realizadas com a $B$. uncinella e suas respectivas identificações. $\mathrm{O} \mathrm{CE}_{50}$ (g DPPH/g extrato) indica a quantidade de extrato necessário para decair a absorbância pela metade de um grama de DPPH em solução. Quanto menor o valor de $\mathrm{CE}_{50}$, maior a atividade antioxidante do extrato.

Tabela 1 - Avaliação da atividade antioxidante e flavonoides dos extratos de Baccharis uncinella obtidos a partir da extração supercrítica.

\begin{tabular}{c|c|c|c|c|c}
\hline Espécie & Solvente & $\mathbf{P}(\mathbf{b a r})$ & $\mathbf{T}\left({ }^{\circ} \mathbf{C}\right)$ & $\mathbf{C E}_{\mathbf{5 0}}$ & \% Flavonoides \\
\hline B. uncinella & $\mathrm{CO}_{2}$ & 150 & 60 & 172,340 & 0,159 \\
\hline B. uncinella & $\mathrm{CO}_{2}$ & 200 & 60 & 177,620 & 0,091 \\
\hline B. uncinella & $\mathrm{CO}_{2}+10 \%$ água & 150 & 60 & 185,285 & 0,037 \\
\hline B. uncinella & $\mathrm{CO}_{2}+10 \%$ água & 200 & 60 & 184,300 & 0,209 \\
\hline B. uncinella & $\mathrm{CO}_{2}+10 \%$ etanol & 150 & 60 & 185,430 & 0,355 \\
\hline B. uncinella & $\mathrm{CO}_{2}+10 \%$ etanol & 200 & 60 & 185,285 & 1,116 \\
\hline
\end{tabular}

Tabela 2 - Avaliação da atividade antioxidante e flavonoides dos extratos de B.uncinella obtidos por maceração com etanol e partição com solventes orgânicos.

\begin{tabular}{c|c|c|c}
\hline Espécie & Tipo de Extrato & $\mathbf{C E}_{\mathbf{5 0}}$ & \% de Flavonoides \\
\hline B. uncinella & extrato bruto (sem partição) & 28,33 & 1,930 \\
\hline B. uncinella & partição com n-hexano & 171,87 & 0,416 \\
\hline B. uncinella & partição com diclorometano & 53,13 & 1,937 \\
\hline B. uncinella & partição com acetato de etila & 11,80 & 5,287 \\
\hline
\end{tabular}

Analisando os resultados, percebeu-se que os extratos supercríticos obtidos nas diferentes condições (Tabela 1) não apresentaram variações significativas em relação à atividade antioxidante. Observou-se que os extratos obtidos sem cossolvente apresentaram maior atividade antioxidante quando comparados aos obtidos com cossolvente, porém esta atividade é baixa quando comparada às atividades das frações polares dos extratos de $B$. uncinella obtidas por maceração seguida de partição (Tabela 2). Com relação à maceração, observou-se que os resultados para a partição utilizando acetato de etila apresentou a maior 


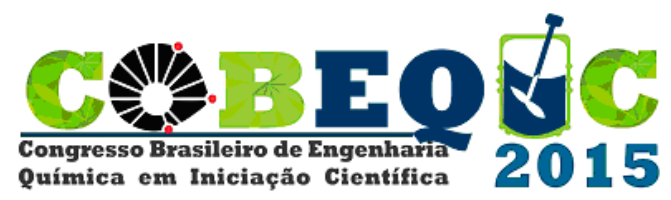

\section{Congresso Brasileiro de Engenharia Química em Iniciação Científica Unicamp - Campinas - SP 19 a 22 de julho de 2015}

atividade antioxidante, isto é, o menor $\mathrm{CE}_{50}$.

Os resultados de concentração de flavonoides variaram de acordo com as condições de extração, o tipo de solvente e o processo extrativo selecionado. O extrato supercrítico com etanol a 200 bar foi o que apresentou o percentual maior entre os extratos supercríticos. $\mathrm{Na}$ maceração, o extrato bruto e o extrato obtido a partir do particionamento com acetato de etila destacaram-se em relação aos demais por apresentarem maiores concentração de flavonoides.

A partir da aplicação do planejamento experimental definido e utilizado foi possível observar que as variáveis avaliadas, tipo de processo extrativo, condições experimentais dos processos extrativos e polaridade dos solventes, têm influência tanto na atividade antioxidante como na concentração de flavonoides dos extratos, com destaque para o teor de flavonoides.

\section{CONCLUSÃO}

O extrato obtido por maceração e particionado com acetato de etila apresentou o melhor potencial de atividade antioxidante entre as amostras avaliadas, assim como o maior percentual de flavonoides. Tal resultado está de acordo com o encontrado na análise por CCD, mostrado na Figura 1. Este fato indica uma seletividade da maceração com metanol seguido de particionamento com acetato de etila para a obtenção de flavonoides a partir de Baccharis uncinella.

\section{REFERENCIAS}

ASCARI, J.; SENS S.L.; NUNES, D.S. Sedative effects of essential oils obtained from Baccharis uncinella. Pharm. Biol., v. 50(1), p.113-119, 2012.

BIESAGA, M. Influence of extraction methods on stability of flavonoids. J. Chromatogr. A, v. 1218(18), p.2505-2512, 2011.

BUDEL, J.M.; DUARTE, M.R.; SANTOS, C.A.M. Progresso da pesquisa sobre o gênero Baccharis, Asteraceae: I - Estudos botânicos. Rev. Bras. Farmacogn., v.15(3), p. 268-271, 2005.

BRAND-WILIAMS, W.; CUVELIER, M.E.; BERSET, C. Use of a free radical method to evaluate antioxidant activity. Food Sci. Technol., v. 28, p. 25-30, 1995.

CASSEL, E.; VARGAS, R.M.F.; BRUN, G.W.; Processos de Extração Supercrítica Aplicados a Produtos Naturais In: Fundamentos de Tecnología de Productos Fitoterapéuticos (2Edn). Porto Alegre: EDIPUCRS, 2008, p. 213-228.

CECHINEL, V., YUNES, R.A. Estratégias para a obtenção de compostos farmacologicamente ativos a partir de plantas medicinais. Conceito sobre modificação estrutural para otimização da atividade. Quim. Nova, v. 21, 1998.

FUNARI, C.S., FERRO, V.O. Análise de própolis. Ciênc. e Tecnol. Aliment. , v. 26(01), p.171-178, 2006.

HUANG, Z.; SHI, X-h.; JIANG, W-j. Theoretical models for supercritical fluid extraction. J. Chromatogr. A, v. 1250, p.2-26, 2012.

OLIVEIRA, S.Q., DAL-PIZZOL, F., GOSMANN, G. Antioxidant activity of Baccharis articulata extracts: isolation of a new compound with antioxidante activity. Free Radical Research, v. 37, p. 555-559, 2003

PIANTINO, C.R.; AQUINO, F.W.B.; FOLLEGATIi-ROMERO, L.A.; CABRAL, F.A. Supercritical $\mathrm{CO}_{2}$ extraction of phenolic compounds from Baccharis dracunculifolia. J. Supercritic. Fluids, v. 47, p. 209$214,2008$. 
XI Congresso Brasileiro de Engenharia Química em Iniciação Científica

VILA, F.C. Identificação dos flavonoides com atividade antioxidante de cana-de-açucar. São Paulo. 2006. 68p. Dissertação (Mestrado em Química Analítica). Instituto de Química, Universidade de São Paulo, Brasil. 